\title{
Электростатическая теория селективности ионообменников
}

\author{
® 2020 Шапошник B.A. \\ Воронежский государственный университет, Воронеж \\ Поступила в редакцию 15.01.2020 г.
}

DOI: $10.17308 /$ sorpchrom.2020.20/2379

Ионообменники являются супрамолекулярными веществами, в которых сочетаются прочные ковалентные связи (hard chemistry) со слабыми ионными и водородными связями (soft chemistry). Ионные связи в ионообменниках ослаблены гидратацией противоионов и фиксированных ионов. Водородные связи образуются между их гидратными молекулами. Элементарный транспортный акт ионного обмена заключается в разрыве слабых связей. Задачей настоящей работы было определение типа связи, определяющей селективность ионообменника.

Для определения энергии ионных связей был применён интегральный вид закона Кулона. Используя допущения Полея были рассчитаны энергии электростатического взаимодействия фиксированных ионов с противоионами. Установлено, что время удерживания катионов щелочных металлов является положительной экспоненциальной функцией энергии энергий электростатического притяжения противоионов фиксированными ионами. Коррекция параметров электростатического взаимодействия ионов была проведена неэмпирическими методами квантовой химии.

Для определения общей энергии разрыва связей противоионов с фиксированными ионами (энергии активации) была измерена температурная зависимость электропроводностей ионообменных мембран контактно-разностным методом. Энергии водородных связей были рассчитаны по разности энергий активации и энергий электростатического притяжения противоионов фиксированными ионами. В результате было установлено, что энергии элементарного транспортного акта в ионообменниках для однозарядных ионов определяются преимущественно водородными связями, а для противоионов более высокого числа зарядов кроме водородных связей существенную роль играют ионные связи, Основным результатом исследования является доказательство определяющей роли электростатического взаимодействия (ионных связей) в избирательности ионообменников.

Ключевые слова: ионный обмен, селективность, электростатические взаимодействия, водородные связи, энергии активации, квантовая химия

\section{Введение}

Понятие селективности было введено в аналитическую химию Т. Бергманом в 1775 г. в его диссертации, которая имела такой резонанс в обществе, что И.В. Гёте написал под её влиянием роман «Избирательное сродство». Тем не менее, Т. Бергману не удалось найти селективные реагенты для каждого аналита, и он предложил метод систематического анализа, при котором смеси веществ разделялись на группы, внутри которых также использовались принципы систематического анализа. Впервые сложные смеси веществ удалось разделить хроматографическим методом, предложенным М.С. Цветом в 1903 г. [1]. Он впервые применил элюентную хроматографию для разделения пигментов растений. Для разделения смесей ионов используется метод высокоэффективной ионообменной хроматографии, которую называют ионной хроматографией [2]. Задачей настоящей работы является развитие электро- 
статической теории селективности ионообменников, которая лежит в основе понимания сущности разделения смесей ионов методом ионной хроматографии.

\section{Кулоновское взаимодействие в ионообменниках}

Первый шаг к созданию электростатической теории ионного обмена, сделал Полей в 1954 г. [3]. Он применил интегральный вид закона Кулона для расчета энергии взаимодействия фиксированных ионов ионообменника с мобильными противоионами

$$
\mathrm{E}=\frac{\left[\mathrm{z}_{+}\right]\left[\mathrm{z}_{-}\right] \mathrm{e}^{2}}{\varepsilon \mathrm{r}},
$$

$z_{+}, z_{-}$- числа зарядов катионов и анионов, $e$ - заряд электрона, $\varepsilon$ - диэлектрическая проницаемость, $\boldsymbol{r}$ - расстояние между центрами положительного и отрицательного зарядов, которые Полей рассматривал как сумму радиусов фиксированного иона и противоиона. Рис. 1 показывает катионную хроматограмму, полученную при разделении катионов щелочных металлов методом двухколоночной хроматографии при использовании в качестве сорбента поверхностно - модифицированного сульфокатионообменника с применением азотнокислого элюента [2]. Как показано на рисунке при разделении катионов щелочных металлов была достигнута высокая степень разрешения хроматографических пиков. Рис. 2 показывает зависимость времен удерживания катионов щелочных металлов и иона аммония от энергии электростатического притяжения фиксированных ионов и противоионов, рассчитанную по радиусам ионов, приведенным в работе Полея [3]. Полученная зависимость показывает, что время удерживания катиона является положительной экспонентой энергии электростатического притяжения катиона фиксированным ионом сульфокатионообменника.

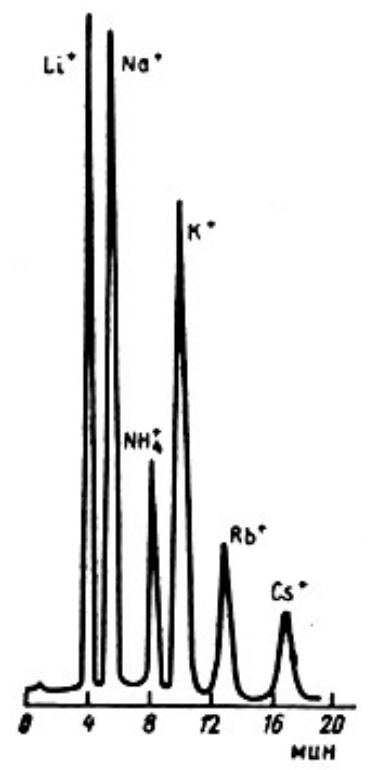

Рис. 1. Хроматограмма, полученная при разделении смеси катионов щелочных металлов и аммония методом двухколоночной ионной хроматографией с применением в качестве элюента азотной кислоты и кондуктометрического детектора [2].

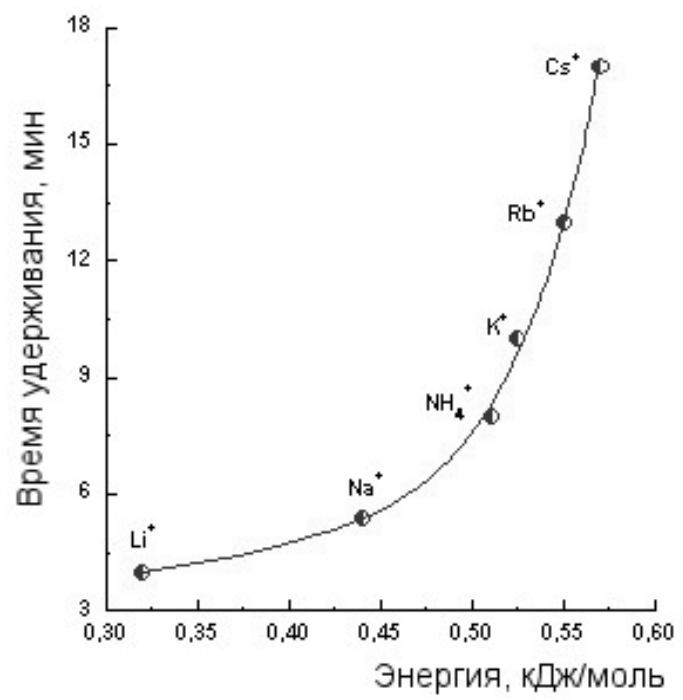

Рис. 2. Зависимость времен удерживания катионов щелочных металлов от энергии электростатического притяжения фиксированных ионов сульфокатионообменника с противоионами. 
Приведенные рисунки позволяют сделать вывод, что порядок выхода ионов из колонки определяет в основном радиусы их ионов. Согласно уравнению Кулона (1) ион, лития имеющий наибольший радиус в гидратированном состоянии, имеет наименьшую энергию взаимодействия, и первым оказывается на выходе из колонки, а ион цезия, который имеет отрицательную гидратацию и, соответственно, большую величину энергию кулоновского взаимодействия оказывается последним из катионов щелочных металлов на выходе из колонки. Полученные рассчитанные величины времён удерживания позволяют предсказать результаты качественного хроматографического анализа без использования стандартных растворов. Недостатком теории было использование суммы радиусов ионов в качестве расстояния между зарядами, целых чисел зарядов ионов и постоянной диэлектрической проницаемости гидратированных ионных пар.

\section{Коррекция электростатической теории методами квантовой химии}

Недостатки электростатической теории могут быть исправлены методами квантовой химии. Нами были рассчитаны структуры катионообменников и анионообменников в формах ионов с разным числом зарядов, используя последовательность расчета структур методами молекулярной механики с неэмпирическими методами квантовой химии, с использованием базисов STO с включением поляризационных функций 6-31G**. Базисный набор с двумя звездочками означает добавление поляризационной $d-G T O$ к каждой $p-$ функции, и добавление $p-G T O$ к $1 s-$ орбиталям атомов водорода. Рис. 3 даёт пример визуализации расчета структуры сульфокатионообменника в форме ионов кальция.

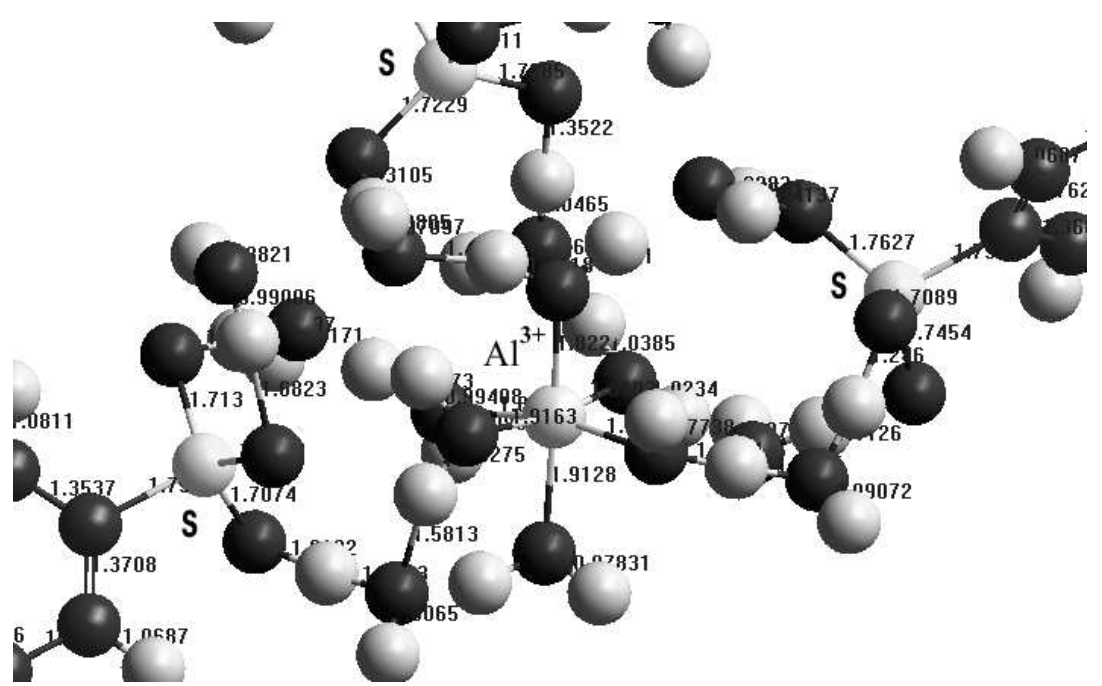

Рис. 3. Оптимизированная структура фрагмента сульфокатионообменника в форме ионов алюминия

Как показывает рисунок 3, противоион алюминия, связан с тремя фиксированными ионами, что увеличивает кулоновское взаимодействие между фиксированными ионами и противоионом. Таблица 1 представляет результаты расчета энергий электростатического взаимодействия для катионообменников и анионообменников в формах противоионов разного заряда. Расстояния между центрами зарядов, рассчитанные методами квантовой химии, меньше суммы радиусов ионов [3]. Диэлектрическая проницаемость, которую Полей принимал постоянной величиной, равной диэлектрической постоянной воды была нами рассчитана по теории Дебая [4], как функция расстояния между центрами зарядов. Рассчитанные методом квантовой хи- 
мии числа зарядов являются дробными величинами и меньшими, чем числа зарядов катионов и анионов вследствие делокализации электронной плотности в молекулах. В результате для противоионов натрия мы получили в 4.3 раза меньшую величину, чем рассчитанная Полеем. Тем не менее, полученная 1.9 кДж/моль в 1.3 раза ниже тепловой энергии при стандартной температуре (2.4 кДж/моль). Означает ли это, что транспорт противоионов натрия в сульфокатионообменнике является безактивационным?

Таблица 1. Результаты расчетов и экспериментов

\begin{tabular}{|c|c|c|c|c|c|}
\hline Противоион & $\mathrm{r}, \AA$ & $\varepsilon$ & $\mathrm{z}$ & $E_{e l}$, кДж/моль & $E_{a}$ кДж/моль \\
\hline $\mathrm{Na}^{+}$ & 4.36 & 17.5 & 0.55 & 1.9 & 21.7 \\
\hline $\mathrm{Ca}^{2+}$ & 4.84 & 20.2 & 0.85 & 9.5 & 27.2 \\
\hline $\mathrm{Al}^{3+}$ & 4.98 & 21.2 & 1.36 & 20.8 & 38.4 \\
\hline $\mathrm{Cl}^{-}$ & 5.36 & 24.8 & 0.73 & 1.2 & 20.4 \\
\hline $\mathrm{SO}_{4}{ }^{2-}$ & 4.80 & 19.9 & 1.01 & 4.6 & 24.6 \\
\hline $\mathrm{PO}_{4}{ }^{3-}$ & 4.51 & 17.9 & 1.23 & 9.9 & 28.8 \\
\hline
\end{tabular}

Для ответа на этот вопрос мы рассчитали энергии активации по результатам температурной зависимости удельной электропроводности ионообменных мембран. Величины удельной электропроводности ионообменных мембран определяли контактно-разностным методом мультимером HIOKI [5]. Мембраны помещали в термостатируемый цилиндр с дистиллированной водой для исключения необменно сорбированных электролитов. Проведя измерения в интервале температур от 20 до $60^{\circ} \mathrm{C}$ мы рассчитали энергии активации $E_{a}$ по уравнению Аррениуса

$$
E_{a}=\frac{R \ln \frac{\kappa_{2}}{\kappa_{1}}}{\left(\frac{1}{T_{1}}-\frac{1}{T_{2}}\right)},
$$

где $\mathrm{R}$ - газовая постоянная, $k$ - удельная электропроводность, $T$ - абсолютная температура. Приведенная в табл. 1 величина экспериментальной энергии активации на порядок выше, чем энергия электростатического притяжения фиксированного иона и противоиона. Разность между ними равна 19.6 кДж/моль, что равно энергии водородной связи, а также энергиям активации вязкого течения воды, самодиффузии молекул воды и их диэлектрической релаксации [6]. Кроме того, эти величины равны энергии перехода фононов деформационных колебаний воды с фундаментального на первый энергетический уровень, которая является минимальной для всех энергий перехода нормальных вод молекул воды [7]

$$
E=h c \sigma_{2}
$$

где $h$ - постоянная Планка, с - скорость света, $\sigma_{2}$ - волновое число деформационных колебаний воды. Малая величина энергии электростатического притяжения фиксированными ионами противоионов в сравнении с энергией активации была объяснена определяющей ролью водородной связи в сравнении с ионной при элементарном акте транспорта однозарядных ионов [8]. Для многозарядных противоионов нельзя делать вывод об определяющей роли водородной связи [9]. Как следует из данных табл. 1 для двухзарядных ионов вклад ионной связи с энергию потенциального барьера, который преодолевает противоион при элементарном акте переноса достаточно велик для вывода об определяющей роли водородной связи, а для трехзарядных ионов энергии водородной и ионной связей близки. 


\title{
Заключение
}

Элементарный акт транспорта при ионном обмене состоит в разрыве ионной и водородной связей, образующихся между фиксированными ионами ионообменника и противоионами, причем вклад водородной связи мало зависит от вида противоиона, а переменная величина электростатического взаимодействия определяет селективность ионообменников.

\section{Список литературы}

1. Цвет М.С.Избранные труды, М., Наука, 2013. $679 \mathrm{c}$.

2. Шпигун О.А., Золотов Ю.А. Ионная хроматография. М. Изд-во МГУ. 1990. 199 с.

3. Pauley J.L. // J.Amer. Chem. Soc. 1954. Vol. 76. pp. 1422-1425.

4. Дебай П. Полярные молекулы. М. Госнаучтехиздат. $1931.247 \mathrm{c}$.

5. Шапошник В.А., Емельянов Д.Е., Дробышева И.В. // Коллоидный журнал. 1984. Т. 46. № 6. С. 1116-1119.
6. Wang J.H., Robinson C.V., Edelman I.S. // J. Amer. Chem. Soc. 1953. Vol. 75. p. 466.

7. Шапошник В.А. // Электрохимия. 1994. T. 30. № 5. C. 638-643.

8. Бутырская Е.В., Шапошник В.А. // Журнал структурной химии. 2003. Т. 44. № 6. С. 1180-1185.

9. Badessa T.S., Shaposhnik V.A. // J. Membrane Science. 2016. Vol. 498. pp. 86-93.

\section{Electrostatic theory of ion exchanger selectivity}

\author{
Shaposhnik Vladimir A. \\ Voronezh State University, Voronezh
}

Ion exchangers are supramolecular substances that combine strong covalent bonds (hard chemistry) with weak ionic and hydrogen bonds (soft chemistry). Ionic bonds in ion exchangers are weakened by the hydration of counter-ions and fixed ions. Hydrogen bonds are formed between their hydrate molecules. The elementary transport act of ion exchange involves the breaking of weak bonds. The purpose of this work was to determine the type of bond that defines the selectivity of ion exchangers.

The integral form of Coulomb's law was used to calculate the energy of ion bonds. Using Pauley's assumptions, the electrostatic interaction energies of fixed ions with counter-ions were calculated. It has been established that the retention time of alkali metal cations is a positive exponential function of the energy of the electrostatic attraction of counter-ions by fixed ions. Correction of the parameters of the electrostatic interaction of ions was performed using non-empirical methods of quantum chemistry.

To determine the total energy of breaking the bonds of counter-ions with fixed ions (activation energy), the temperature dependence of the electrical conductivity of ion-exchange membranes was measured using the contact-difference method. The hydrogen bond energies were calculated from the difference between the activation energies and the electrostatic attraction energies of counter-ions by fixed ions. As a result, it was found that the energies of the elementary transport act in ion exchangers for single-charged ions are determined mainly by hydrogen bonds, while ion bonds, in addition to hydrogen bonds, play a significant role for counter-ions of a higher charge number. The main result of the study is confirming the determining role of the electrostatic interaction (ionic bonds) in the selectivity of ion exchangers.

Keywords: ion exchange, selectivity, electrostatic interactions, hydrogen bonds, energy of activation, quantum chemistry

\section{References}

1. Tswett M., Selected Works, M., Science, 2013. $679 \mathrm{p}$.
2. Shpigun O.A., Zolotov Yu.A., Ionic Chromatography, Moscow Univ., 1990, 199 p. 
3. Pauley J.L., J.Amer. Chem. Soc., 1954, Vol. 76, pp. 1422-1425.

4. Debye P., Polar molecules, N.Y., 1929. $172 \mathrm{p}$.

5. Shaposhnik V.A., Emel'anov D.E., Drobysheva I.V., Colloid. J., 1984, Vol. 46, No 6, pp. 1116-1119.

6. Wang J.H., Robinson C.V., Edelman I.S., J. Amer. Chem. Soc., 1953, Vol. 75, p. 466.

\section{Шапошник Владимир Алексеевич -} д.Х.н., профессор кафедры аналитической химии Воронежского госуниверситета, Воронеж
7. Shaposhnik V.A., Rus. J. Electrochem., 1994, Vol.30, No 5, pp. 638-643.

8. Butyrskaya E.V., Shaposhnik V.A., J. Structural Chem., 2003, Vol. 44, No 6, pp. 1180-1185.

9. Badessa T.S., Shaposhnik V.A., J. Membrane Science, 2016, Vol. 498, pp. 86-93.

Shaposhnik Vladimir A. - Dr. Sc., Professor, Department of Analytical Chemistry Voronezh State University, Voronezh, e-mail: 